\title{
Modified Mann-Halpern Algorithms for Pseudocontractive Mappings
}

\author{
Hong-bo Du \\ School of Science, Shenyang University of Technology, Shenyang 110178, China \\ Correspondence should be addressed to Hong-bo Du; hb_du@163.com \\ Received 18 February 2013; Accepted 20 March 2013 \\ Academic Editor: Yisheng Song
}

Copyright (C) 2013 Hong-bo Du. This is an open access article distributed under the Creative Commons Attribution License, which permits unrestricted use, distribution, and reproduction in any medium, provided the original work is properly cited.

Modified Mann-Halpern algorithms for finding the fixed points of pseudocontractive mappings are presented. Strong convergence theorems are obtained.

\section{Introduction}

Finding the fixed points of nonlinear operators is an important topic in fixed point theory, due to the fact that many nonlinear problems can be reformulated as fixed point equations of nonlinear mappings. The research of this area dates back to Picard's and Banach's time. Now it is well known that the Picard iterates $\left\{T^{n} x\right\}$ converge to the unique fixed point of $T$ whenever $T$ is a contraction of a complete metric space. However, if $T$ is not a contraction (e.g., nonexpansive), then the Picard algorithm $\left\{T^{n} x\right\}$ does not converge. Consequently, Mann's algorithm was constructed by Mann [1] in 1953:

$$
x^{n+1}=\left(1-\alpha_{n}\right) x^{n}+\alpha_{n} T x^{n}, \quad n \geq 0 .
$$

There are a large number of papers on Mann's algorithm in the literature. See [2-5]. Now we know that if $T$ is nonexpansive, then Mann's algorithm converges weakly to a fixed point of $T$. This algorithm however does not converge in the strong topology.

In order to get the strong convergence, the following Halpern's algorithm was introduced:

$$
x^{n+1}=\alpha_{n} u+\left(1-\alpha_{n}\right) T x^{n}, \quad n \geq 0 .
$$

The interest and importance of Halpern iterative method lie in the fact that strong convergence of the sequence $\left\{x_{n}\right\}$ is achieved under certain mild conditions on parameter $\left\{\alpha_{n}\right\}$ in a general Banach space. Please refer to [6-12].
In the present paper, we are devoted to find the fixed points of pseudocontractive mappings. For some related works, please see [13-23]. The interest of pseudocontractions lies in their connection with monotone operators. Browder and Petryshyn [24] studied weak convergence of Mann's algorithm for the class of strict pseudocontractions. But Mann's algorithm fails to converge for Lipschitzian pseudocontractions [25].

Inspired by the results in the literature, the main purpose of this paper is to construct an iterative method for finding the fixed points of pseudocontractive mappings. Under some mild conditions, strong convergence results are given.

\section{Preliminaries}

Let $H$ be a real Hilbert space with inner product $\langle\cdot, \cdot\rangle$ and norm $\|\cdot\|$, respectively. Let $C$ be a nonempty closed convex subset of $H$. A mapping $T: C \rightarrow C$ is called pseudocontractive if

$$
\langle T x-T y, x-y\rangle \leq\|x-y\|^{2}, \quad x, y \in C .
$$

A mapping $T: C \rightarrow C$ is called $k$-Lipschitzian if there exists $k>0$ such that

$$
\|T x-T y\| \leq k\|x-y\|,
$$

for all $x, y \in C$. In this case, if $k<1$, then $T$ is a $k$-contraction. 
It is well known that in a real Hilbert space $H$ the following inequality holds:

$$
\|x+y\|^{2} \leq\|x\|^{2}+2\langle y, x+y\rangle,
$$

for all $x, y \in H$.

In the present paper, we will use the following notations:

(i) we use $\operatorname{Fix}(T)$ to denote the set of fixed points of $T$;

(ii) $x_{n} \rightarrow x$ denotes the weak convergence of $x_{n}$ to $x$;

(iii) $x_{n} \rightarrow x$ denotes the strong convergence of $x_{n}$ to $x$.

Lemma 1 (see [26]). Let $C$ be a closed convex subset of a real Hilbert space $H$. Let $T: C \rightarrow C$ be a continuous pseudocontractive mapping. Then $(I-T)$ is demiclosed at zero.

Lemma 2 (see [27]). Let $\left\{w_{n}\right\}$ be a sequence of real numbers. Assume $\left\{w_{n}\right\}$ does not decrease at infinity; that is, there exists at least a subsequence $\left\{w_{n_{k}}\right\}$ of $\left\{w_{n}\right\}$ such that $w_{n_{k}} \leq w_{n_{k}+1}$ for all $k \geq 0$. For every $n \geq M$, define an integer sequence $\{W(n)\}$ as

$$
W(n)=\max \left\{i \leq n: w_{n_{i}}<w_{n_{i}+1}\right\} .
$$

Then $W(n) \rightarrow \infty$ as $n \rightarrow \infty$ and for all $n \geq M$

$$
\max \left\{w_{W(n)}, w_{n}\right\} \leq w_{W(n)+1} .
$$

Lemma 3 (see [28]). Assume that $\left\{A_{n}\right\}$ is a sequence of nonnegative real numbers such that

$$
A_{n+1} \leq\left(1-\gamma_{n}\right) A_{n}+\xi_{n}
$$

where $\left\{\gamma_{n}\right\}$ is a sequence in $(0,1)$ and $\left\{\xi_{n}\right\}$ is a sequence such that

(1) $\sum_{n=1}^{\infty} \gamma_{n}=\infty$;

(2) $\lim \sup _{n \rightarrow \infty}\left(\xi_{n} / \gamma_{n}\right) \leq 0$ or $\sum_{n=1}^{\infty}\left|\xi_{n}\right|<\infty$.

Then $\lim _{n \rightarrow \infty} A_{n}=0$.

\section{Main Results}

Now we present the statement of our algorithm.

The Modified Mann-Halpern Algorithm. Let $C$ be a nonempty closed convex subset of a real Hilbert space $H$. Let $T: C \rightarrow C$ be a pseudocontractive mapping and $S: C \rightarrow H$ a $\rho$ contractive mapping. Let $\left\{\alpha_{n}\right\},\left\{\beta_{n}\right\}$, and $\left\{\gamma_{n}\right\}$ be three real number sequences in $[0,1]$. We have the following steps.

(1) Initialization:

$$
\forall x_{0} \in C
$$

(2) Mann step: for a given $x_{n}$, define a sequence $y_{n}$ by

$$
y_{n}=\left(1-\gamma_{n}\right) x_{n}+\gamma_{n} T x_{n}
$$

for all $n \geq 0$.
(3) Halpern step: for a given $x_{n}$ and $y_{n}$, define

$$
x_{n+1}=\alpha_{n} S\left(x_{n}\right)+\left(1-\alpha_{n}-\beta_{n}\right) x_{n}+\beta_{n} T y_{n},
$$

for all $n \geq 0$.

In the following, we assume that

(i) the mapping $T: C \rightarrow C$ is $k$-Lipschitzian;

(ii) the sequences $\left\{\alpha_{n}\right\},\left\{\beta_{n}\right\}$, and $\left\{\gamma_{n}\right\}$ satisfy the following conditions $(\mathrm{C} 1)-(\mathrm{C} 5)$ :

$$
\begin{aligned}
& \text { (C1): } \lim _{n \rightarrow \infty} \alpha_{n}=0 ; \\
& \text { (C2): } \sum_{n=1}^{\infty} \alpha_{n}=\infty ; \\
& \text { (C3): } \alpha_{n}+\beta_{n} \leq \gamma_{n} ; \\
& \text { (C4): } 0<\liminf _{n \rightarrow \infty} \beta_{n} ; \\
& \text { (C5): } 0<\lim _{n \rightarrow \infty} \sup _{n \rightarrow \infty}<1 /\left(\sqrt{1+k^{2}}+1\right) .
\end{aligned}
$$

Now, we prove our main result as follows.

Theorem 4. Suppose $\operatorname{Fix}(T) \neq \emptyset$. Then the sequence $\left\{x_{n}\right\}$ defined by (11) converges strongly to a fixed point of $T$.

Proof. Since $S$ is a $\rho$-condition, then $\operatorname{Proj}_{\text {Fix }(T)} S$ is a contractive mapping (where Proj is the metric projection). Hence, there exists a unique $u$ such that $u=\operatorname{Proj}_{\operatorname{Fix}(T)} S(u)$. In the sequel, we will show that the sequence $\left\{x_{n}\right\}$ defined by (11) converges strongly to $u$.

From (11), we get

$$
\begin{aligned}
& \left\|x_{n+1}-u\right\| \\
& =\left\|\alpha_{n} S\left(x_{n}\right)+\left(1-\alpha_{n}-\beta_{n}\right) x_{n}+\beta_{n} T y_{n}-u\right\| \\
& \leq\left\|\alpha_{n}\left(S\left(x_{n}\right)-u\right)+\left(1-\alpha_{n}-\beta_{n}\right)\left(x_{n}-u\right)+\beta_{n}\left(T y_{n}-u\right)\right\| \\
& =\| \alpha_{n}\left(S\left(x_{n}\right)-u\right)+\left(1-\alpha_{n}\right) \\
& \quad \times\left(\frac{1-\alpha_{n}-\beta_{n}}{1-\alpha_{n}}\left(x_{n}-u\right)+\frac{\beta_{n}}{1-\alpha_{n}}\left(T y_{n}-u\right)\right) \| \\
& \leq \alpha_{n}\left\|S\left(x_{n}\right)-u\right\|+\left(1-\alpha_{n}\right) \\
& \quad \times\left\|\frac{\left(1-\alpha_{n}-\beta_{n}\right)\left(x_{n}-u\right)}{1-\alpha_{n}}+\frac{\beta_{n}\left(T y_{n}-u\right)}{1-\alpha_{n}}\right\| .
\end{aligned}
$$

It is well known that there holds the following inequality in Hilbert spaces:

$$
\begin{aligned}
\|t x+(1-t) y\|^{2}= & t\|x\|^{2}+(1-t)\|y\|^{2} \\
& -t(1-t)\|x-y\|^{2}
\end{aligned}
$$

for all $x, y \in H$ and $t \in[0,1]$. Hence, we have

$$
\begin{gathered}
\left\|\frac{\left(1-\alpha_{n}-\beta_{n}\right)\left(x_{n}-u\right)}{1-\alpha_{n}}+\frac{\beta_{n}\left(T y_{n}-u\right)}{1-\alpha_{n}}\right\|^{2} \\
\leq \frac{1-\alpha_{n}-\beta_{n}}{1-\alpha_{n}}\left\|x_{n}-u\right\|^{2}+\frac{\beta_{n}}{1-\alpha_{n}}\left\|T y_{n}-u\right\|^{2} \\
-\frac{\beta_{n}\left(1-\alpha_{n}-\beta_{n}\right)}{\left(1-\alpha_{n}\right)^{2}}\left\|x_{n}-T y_{n}\right\|^{2} .
\end{gathered}
$$


We know that $T$ is pseudocontractive if and only if $T$ satisfies the condition

$$
\|T x-T y\|^{2} \leq\|x-y\|^{2}+\|(I-T) x-(I-T) y\|^{2}
$$

for all $x, y \in C$. Since $u \in \operatorname{Fix}(T)$, we have from (15) that

$$
\|T x-u\|^{2} \leq\|x-u\|^{2}+\|x-T x\|^{2},
$$

for all $x \in C$.

By using (13) and (16), we obtain

$$
\begin{aligned}
\| T y_{n}- & u \|^{2} \\
\leq & \left\|y_{n}-u\right\|^{2}+\left\|y_{n}-T y_{n}\right\|^{2} \\
= & \left\|\left(1-\gamma_{n}\right) x_{n}+\gamma_{n} T x_{n}-u\right\|^{2} \\
& +\left\|\left(1-\gamma_{n}\right) x_{n}+\gamma_{n} T x_{n}-T y_{n}\right\|^{2} \\
= & \left\|\left(1-\gamma_{n}\right)\left(x_{n}-u\right)+\gamma_{n}\left(T x_{n}-u\right)\right\|^{2} \\
& +\left\|\left(1-\gamma_{n}\right)\left(x_{n}-T y_{n}\right)+\gamma_{n}\left(T x_{n}-T y_{n}\right)\right\|^{2} \\
= & \left(1-\gamma_{n}\right)\left\|x_{n}-u\right\|^{2}+\gamma_{n}\left\|T x_{n}-u\right\|^{2} \\
& -\gamma_{n}\left(1-\gamma_{n}\right)\left\|x_{n}-T x_{n}\right\|^{2} \\
& +\left(1-\gamma_{n}\right)\left\|x_{n}-T y_{n}\right\|^{2}+\gamma_{n}\left\|T x_{n}-T y_{n}\right\|^{2} \\
& -\gamma_{n}\left(1-\gamma_{n}\right)\left\|x_{n}-T x_{n}\right\|^{2} \\
\leq & \left(1-\gamma_{n}\right)\left\|x_{n}-u\right\|^{2} \\
& +\gamma_{n}\left(\left\|x_{n}-u\right\|^{2}+\left\|x_{n}-T x_{n}\right\|^{2}\right) \\
& -\gamma_{n}\left(1-\gamma_{n}\right)\left\|x_{n}-T x_{n}\right\|^{2} \\
& +\left(1-\gamma_{n}\right)\left\|x_{n}-T y_{n}\right\|^{2}+\gamma_{n}\left\|T x_{n}-T y_{n}\right\|^{2} \\
& -\gamma_{n}\left(1-\gamma_{n}\right)\left\|x_{n}-T x_{n}\right\|^{2} . \\
&
\end{aligned}
$$

Note that $T$ is $k$-Lipschitzian and

$$
\left\|x_{n}-y_{n}\right\|=\gamma_{n}\left\|x_{n}-T x_{n}\right\| .
$$

From (17), we have

$$
\begin{aligned}
\| T y_{n}- & u \|^{2} \\
\leq & \left(1-\gamma_{n}\right)\left\|x_{n}-u\right\|^{2} \\
& +\gamma_{n}\left(\left\|x_{n}-u\right\|^{2}+\left\|x_{n}-T x_{n}\right\|^{2}\right) \\
& -\gamma_{n}\left(1-\gamma_{n}\right)\left\|x_{n}-T x_{n}\right\|^{2} \\
& +\left(1-\gamma_{n}\right)\left\|x_{n}-T y_{n}\right\|^{2}+\gamma_{n} k^{2}\left\|x_{n}-y_{n}\right\|^{2} \\
& -\gamma_{n}\left(1-\gamma_{n}\right)\left\|x_{n}-T x_{n}\right\|^{2} \\
= & \left(1-\gamma_{n}\right)\left\|x_{n}-u\right\|^{2} \\
& +\gamma_{n}\left(\left\|x_{n}-u\right\|^{2}+\left\|x_{n}-T x_{n}\right\|^{2}\right) \\
& -\gamma_{n}\left(1-\gamma_{n}\right)\left\|x_{n}-T x_{n}\right\|^{2} \\
& +\left(1-\gamma_{n}\right)\left\|x_{n}-T y_{n}\right\|^{2}+\gamma_{n}^{3} k^{2}\left\|x_{n}-T x_{n}\right\|^{2} \\
& -\gamma_{n}\left(1-\gamma_{n}\right)\left\|x_{n}-T x_{n}\right\|^{2} \\
= & \left\|x_{n}-u\right\|^{2}+\left(1-\gamma_{n}\right)\left\|x_{n}-T y_{n}\right\|^{2} \\
& -\gamma_{n}\left(1-2 \gamma_{n}-\gamma_{n}^{2} k^{2}\right)\left\|x_{n}-T x_{n}\right\|^{2} .
\end{aligned}
$$

By condition (C5), without loss of generality, we may assume that $\gamma_{n} \leq a<1 /\left(\sqrt{1+k^{2}}+1\right)$ for all $n$. Then, we have $1-$ $2 \gamma_{n}-\gamma_{n}^{2} L^{2}>0$ for all $n \geq 0$. Substituting (19) to (14) and noting condition (C3), we have

$$
\begin{gathered}
\left\|\frac{\left(1-\alpha_{n}-\beta_{n}\right)\left(x_{n}-u\right)}{1-\alpha_{n}}+\frac{\beta_{n}\left(T y_{n}-u\right)}{1-\alpha_{n}}\right\|^{2} \\
\leq \frac{1-\alpha_{n}-\beta_{n}}{1-\alpha_{n}}\left\|x_{n}-u\right\|^{2}+\frac{\beta_{n}}{1-\alpha_{n}} \\
\quad \times\left(\left\|x_{n}-u\right\|^{2}+\left(1-\gamma_{n}\right)\left\|x_{n}-T y_{n}\right\|^{2}\right) \\
\quad-\frac{\beta_{n}\left(1-\alpha_{n}-\beta_{n}\right)}{\left(1-\alpha_{n}\right)^{2}}\left\|x_{n}-T y_{n}\right\|^{2} \\
=\left\|x_{n}-u\right\|^{2}+\frac{\beta_{n}\left(\alpha_{n}+\beta_{n}-\gamma_{n}\right)}{\left(1-\alpha_{n}\right)^{2}}\left\|x_{n}-T y_{n}\right\|^{2} \\
\leq\left\|x_{n}-u\right\|^{2} .
\end{gathered}
$$

Therefore,

$$
\left\|\frac{\left(1-\alpha_{n}-\beta_{n}\right)\left(x_{n}-u\right)}{1-\alpha_{n}}+\frac{\beta_{n}\left(T y_{n}-u\right)}{1-\alpha_{n}}\right\| \leq\left\|x_{n}-u\right\| .
$$


It follows from (12) and (21) that

$$
\begin{aligned}
\left\|x_{n+1}-u\right\| & \\
\leq & \alpha_{n}\left\|S\left(x_{n}\right)-u\right\|+\left(1-\alpha_{n}\right)\left\|x_{n}-u\right\| \\
\leq & \alpha_{n}\left\|S\left(x_{n}\right)-S(u)\right\|+\alpha_{n}\|S(u)-u\| \\
& +\left(1-\alpha_{n}\right)\left\|x_{n}-u\right\| \\
\leq & \alpha_{n} \rho\left\|x_{n}-u\right\|+\alpha_{n}\|S(u)-u\| \\
& +\left(1-\alpha_{n}\right)\left\|x_{n}-u\right\| \\
= & \alpha_{n}\|S(u)-u\|+\left[1-(1-\rho) \alpha_{n}\right]\left\|x_{n}-u\right\| \\
\leq & \max \left\{\left\|x_{n}-u\right\|, \frac{\|S(u)-u\|}{1-\rho}\right\} \\
\leq & \max \left\{\left\|x_{0}-u\right\|, \frac{\|S(u)-u\|}{1-\rho}\right\} .
\end{aligned}
$$

This implies that the sequence $\left\{x_{n}\right\}$ is bounded.

From (5) and (11), we have

$$
\begin{aligned}
\| x_{n+1}- & u \|^{2} \\
= & \|\left(1-\alpha_{n}\right)\left(x_{n}-u\right)-\beta_{n}\left(x_{n}-T y_{n}\right) \\
& +\alpha_{n}\left(S\left(x_{n}\right)-u\right) \|^{2} \\
\leq & \left\|\left(1-\alpha_{n}\right)\left(x_{n}-u\right)-\beta_{n}\left(x_{n}-T y_{n}\right)\right\|^{2} \\
& +2 \alpha_{n}\left\langle S\left(x_{n}\right)-u, x_{n+1}-u\right\rangle \\
= & \left\|\left(1-\alpha_{n}\right)\left(x_{n}-u\right)\right\|^{2} \\
& -2 \beta_{n}\left(1-\alpha_{n}\right)\left\langle x_{n}-T y_{n}, x_{n}-u\right\rangle \\
& +\beta_{n}^{2}\left\|x_{n}-T y_{n}\right\|^{2} \\
& +2 \alpha_{n}\left\langle S\left(x_{n}\right)-u, x_{n+1}-u\right\rangle .
\end{aligned}
$$

Note that (19) is equivalent to

$$
\begin{aligned}
& 2\left\langle x_{n}-T y_{n}, x_{n}-u\right\rangle \\
& \geq \gamma_{n}\left\|x_{n}-T y_{n}\right\|^{2} \\
& \quad+\gamma_{n}\left(1-2 \gamma_{n}-\gamma_{n}^{2} k^{2}\right)\left\|x_{n}-T x_{n}\right\|^{2} .
\end{aligned}
$$

Therefore,

$$
\begin{aligned}
\| x_{n+1}- & u \|^{2} \\
\leq & \left(1-\alpha_{n}\right)\left\|x_{n}-u\right\|^{2} \\
& -\beta_{n}\left(1-\alpha_{n}\right) \gamma_{n}\left\|x_{n}-T y_{n}\right\|^{2} \\
& +\beta_{n}^{2}\left\|x_{n}-T y_{n}\right\|^{2} \\
& -\beta_{n}\left(1-\alpha_{n}\right) \gamma_{n}\left(1-2 \gamma_{n}-\gamma_{n}^{2} k^{2}\right)\left\|x_{n}-T x_{n}\right\|^{2} \\
& +2 \alpha_{n}\left\langle S\left(x_{n}\right)-u, x_{n+1}-u\right\rangle \\
\leq & \left(1-\alpha_{n}\right)\left\|x_{n}-u\right\|^{2} \\
& -\beta_{n}\left(1-\alpha_{n}\right) \gamma_{n}\left(1-2 \gamma_{n}-\gamma_{n}^{2} k^{2}\right)\left\|x_{n}-T x_{n}\right\|^{2} \\
& +2 \alpha_{n}\left\langle S\left(x_{n}\right)-u, x_{n+1}-u\right\rangle .
\end{aligned}
$$

It follows that

$$
\begin{aligned}
& \left\|x_{n+1}-u\right\|^{2}-\left\|x_{n}-u\right\|^{2} \\
& +\beta_{n}\left(1-\alpha_{n}\right) \gamma_{n}\left(1-2 \gamma_{n}-\gamma_{n}^{2} k^{2}\right)\left\|x_{n}-T x_{n}\right\|^{2} \\
& \quad \leq \alpha_{n}\left(\left\langle 2 S\left(x_{n}\right)-u, x_{n+1}-u\right\rangle-\left\|x_{n}-u\right\|^{2}\right) .
\end{aligned}
$$

Since $x_{n}$ and $S\left(x_{n}\right)$ are bounded, there exists $M>0$ such that $\sup _{n}\left\{2\left\langle S\left(x_{n}\right)-u, x_{n+1}-u\right\rangle-\left\|x_{n}-u\right\|^{2}\right\} \leq M$. So

$$
\begin{aligned}
& \left\|x_{n+1}-u\right\|^{2}-\left\|x_{n}-u\right\|^{2} \\
& +\beta_{n}\left(1-\alpha_{n}\right) \gamma_{n}\left(1-2 \gamma_{n}-\gamma_{n}^{2} k^{2}\right)\left\|x_{n}-T x_{n}\right\|^{2} \\
& \quad \leq \alpha_{n} M .
\end{aligned}
$$

Next, we prove two cases.

Assume there exists an integer $m>0$ such that $\left\{\left\|x_{n}-u\right\|\right\}$ is decreasing for all $n \geq m$.

In this case, we know that $\lim _{n \rightarrow \infty}\left\|x_{n}-u\right\|$ exists. From (27), we deduce

$$
\begin{gathered}
\beta_{n}\left(1-\alpha_{n}\right) \gamma_{n}\left(1-2 \gamma_{n}-\gamma_{n}^{2} k^{2}\right)\left\|x_{n}-T x_{n}\right\|^{2} \\
\leq\left\|x_{n}-u\right\|^{2}-\left\|x_{n+1}-u\right\|^{2}+M \alpha_{n} .
\end{gathered}
$$

By conditions (C4) and (C5), we have $\liminf _{n \rightarrow \infty} \beta_{n}(1-$ $\left.\alpha_{n}\right) \gamma_{n}\left(1-2 \gamma_{n}-\gamma_{n}^{2} k^{2}\right)>0$. Thus, from (28), we get

$$
\lim _{n \rightarrow \infty}\left\|x_{n}-T x_{n}\right\|=0 \text {. }
$$

Since $\left\{x_{n}\right\}$ is bounded, there exists a subsequence $\left\{x_{n_{k}}\right\}$ of $\left\{x_{n}\right\}$ satisfying

$$
\begin{gathered}
x_{n_{k}} \longrightarrow \tilde{x} \in C, \\
\limsup _{n \rightarrow \infty}\left\langle S(u)-u, x_{n}-u\right\rangle=\lim _{k \rightarrow \infty}\left\langle S(u)-u, x_{n_{k}}-u\right\rangle .
\end{gathered}
$$

Thus, we use the demiclosed principle of $T$ (Lemma 1) to deduce

$$
\tilde{x} \in \operatorname{Fix}(T) .
$$


So

$$
\begin{aligned}
\limsup _{n \rightarrow \infty}\left\langle S(u)-u, x_{n}-u\right\rangle & =\lim _{k \rightarrow \infty}\left\langle S(u)-u, x_{n_{k}}-u\right\rangle \\
& =\langle S(u)-u, \tilde{x}-u\rangle \\
& \leq 0 .
\end{aligned}
$$

Returning to (25) and using (5) we obtain

$$
\begin{aligned}
\| x_{n+1}- & u \|^{2} \\
\leq & \left(1-\alpha_{n}\right)\left\|x_{n}-u\right\|^{2} \\
& +2 \alpha_{n}\left\langle S\left(x_{n}\right)-u, x_{n+1}-u\right\rangle \\
= & \left(1-\alpha_{n}\right)\left\|x_{n}-u\right\|^{2} \\
& +2 \alpha_{n}\left\langle S\left(x_{n}\right)-S(u), x_{n+1}-u\right\rangle \\
& +2 \alpha_{n}\left\langle S(u)-u, x_{n+1}-u\right\rangle \\
\leq & \left(1-\alpha_{n}\right)\left\|x_{n}-u\right\|^{2} \\
& +2 \alpha_{n} \rho\left\|x_{n}-u\right\|\left\|x_{n+1}-u\right\| \\
& +2 \alpha_{n}\left\langle S(u)-u, x_{n+1}-u\right\rangle \\
\leq & \left(1-\alpha_{n}\right)\left\|x_{n}-u\right\|^{2} \\
& +\alpha_{n} \rho\left(\left\|x_{n}-u\right\|^{2}+\left\|x_{n+1}-u\right\|^{2}\right) \\
& +2 \alpha_{n}\left\langle S(u)-u, x_{n+1}-u\right\rangle .
\end{aligned}
$$

It follows that

$$
\begin{aligned}
\left\|x_{n+1}-u\right\|^{2} \leq & {\left[1-(1-\rho) \alpha_{n}\right]\left\|x_{n}-u\right\|^{2} } \\
& +\frac{2 \alpha_{n}}{1-\alpha_{n} \rho}\left\langle S u-u, x_{n+1}-u\right\rangle .
\end{aligned}
$$

In Lemma 3, we take $A_{n}=\left\|x_{n+1}-u\right\|^{2}, \gamma_{n}=(1-\rho) \alpha_{n}$, and $\xi_{n}=\left(2 \alpha_{n} /\left(1-\alpha_{n} \rho\right)\right)\left\langle S u-u, x_{n+1}-u\right\rangle$. We can check easily that $\sum_{n=1}^{\infty} \gamma_{n}=\infty$ and $\lim \sup _{n \rightarrow \infty}\left(\xi_{n} / \gamma_{n}\right) \leq 0$. Thus, we deduce that $x_{n} \rightarrow u$.

Assume there exists an integer $n_{0}$ such that $\left\|x_{n_{0}}-u\right\| \leq$ $\left\|x_{n_{0}+1}-u\right\|$. In this case, we set $\omega_{n}=\left\{\left\|x_{n}-u\right\|\right\}$. Then, we have $\omega_{n_{0}} \leq \omega_{n_{0}+1}$. Define an integer sequence $\left\{W_{n}\right\}$ for all $n \geq n_{0}$ as follows:

$$
W(n)=\max \left\{l \in \mathbb{N} \mid n_{0} \leq l \leq n, \omega_{l} \leq \omega_{l+1}\right\} .
$$

It is clear that $W(n)$ is a nondecreasing sequence satisfying

$$
\begin{gathered}
\lim _{n \rightarrow \infty} W(n)=\infty, \\
\omega_{\tau(n)} \leq \omega_{W(n)+1},
\end{gathered}
$$

for all $n \geq n_{0}$. From (28), we get

$$
\lim _{n \rightarrow \infty}\left\|x_{W(n)}-T x_{W(n)}\right\|=0 .
$$

This implies that $\omega_{w}\left(x_{W(n)}\right) \subset \operatorname{Fix}(T)$. Thus, we obtain

$$
\limsup _{n \rightarrow \infty}\left\langle S(u)-u, x_{W(n)}-u\right\rangle \leq 0 .
$$

Since $\omega_{W(n)} \leq \omega_{W(n)+1}$, we have from (34) that

$$
\begin{aligned}
\omega_{W(n)}^{2} \leq & \omega_{W(n)+1}^{2} \\
\leq & {\left[1-(1-\rho) \alpha_{W}(n)\right] \omega_{W(n)}^{2} } \\
& +\frac{2 \alpha_{W}(n)}{1-\alpha_{W}(n) \rho}\left\langle S u-u, x_{W(n)+1}-u\right\rangle .
\end{aligned}
$$

It follows that

$$
\omega_{W(n)}^{2} \leq \frac{2}{\left(1-\alpha_{W}(n) \rho\right)(1-\rho)}\left\langle S u-u, x_{W(n)+1}-u\right\rangle .
$$

Combining (38) and (40), we have

$$
\limsup _{n \rightarrow \infty} \omega_{W(n)} \leq 0
$$

and hence

$$
\lim _{n \rightarrow \infty} \omega_{W(n)}=0
$$

From (34), we obtain

$$
\begin{aligned}
\left\|x_{W(n)+1}-u\right\|^{2} & \\
\leq & {\left[1-(1-\rho) \alpha_{W}(n)\right]\left\|x_{W}(n)-u\right\|^{2} } \\
& +\frac{2 \alpha_{W}(n)}{1-\alpha_{W}(n) \rho}\left\langle S u-u, x_{W(n)+1}-u\right\rangle .
\end{aligned}
$$

It follows that

$$
\limsup _{n \rightarrow \infty} \omega_{W(n)+1} \leq \lim _{n \rightarrow \infty} \sup _{W(n)} \omega
$$

This together with (42) implies that

$$
\lim _{n \rightarrow \infty} \omega_{W(n)+1}=0
$$

Applying Lemma 2 we get

$$
0 \leq \omega_{n} \leq \max \left\{\omega_{W(n)}, \omega_{W(n)+1}\right\} .
$$

Therefore, $\omega_{n} \rightarrow 0$. That is, $x_{n} \rightarrow u$. The proof is completed.

\section{Conclusions}

It is now well known that Mann's algorithm fails to converge for Lipschitzian pseudocontractions. Strong convergence of Ishikawa's algorithm has not been achieved without compactness assumption. In the present paper, modified MannHalpern algorithms for finding the fixed points of pseudocontractive mappings are presented. Strong convergence theorems are obtained. 


\section{References}

[1] W. R. Mann, "Mean value methods in iteration," Proceedings of the American Mathematical Society, vol. 4, pp. 506-510, 1953.

[2] A. Moudafi, "Krasnoselski-Mann iteration for hierarchical fixed-point problems," Inverse Problems, vol. 23, no. 4, pp. 1635$1640,2007$.

[3] S. Reich, "Weak convergence theorems for nonexpansive mappings in Banach spaces," Journal of Mathematical Analysis and Applications, vol. 67, no. 2, pp. 274-276, 1979.

[4] T. H. Kim and H. K. Xu, "Robustness of Mann's algorithm for nonexpansive mappings," Journal of Mathematical Analysis and Applications, vol. 327, no. 2, pp. 1105-1115, 2007.

[5] Y. Yao, R. Chen, and J.-C. Yao, "Strong convergence and certain control conditions for modified Mann iteration," Nonlinear Analysis. Theory, Methods \& Applications A. Theory and Methods, vol. 68, no. 6, pp. 1687-1693, 2008.

[6] T. Suzuki, "Strong convergence of approximated sequences for nonexpansive mappings in Banach spaces," Proceedings of the American Mathematical Society, vol. 135, pp. 99-106, 2007.

[7] P.-E. Maingé, "Approximation methods for common fixed points of nonexpansive mappings in Hilbert spaces," Journal of Mathematical Analysis and Applications, vol. 325, no. 1, pp. 469479, 2007.

[8] N. Shioji and W. Takahashi, "Strong convergence of approximated sequences for nonexpansive mappings in Banach spaces," Proceedings of the American Mathematical Society, vol. 125, no. 12, pp. 3641-3645, 1997.

[9] H. K. Xu, "Viscosity approximation methods for nonexpansive mappings," Journal of Mathematical Analysis and Applications, vol. 298, no. 1, pp. 279-291, 2004.

[10] G. Lopez, V. Martin, and H.-K. Xu, "Perturbation techniques for nonexpansive mappings with applications," Nonlinear Analysis. Real World Applications, vol. 10, no. 4, pp. 2369-2383, 2009.

[11] G. Marino and H.-K. Xu, "A general iterative method for nonexpansive mappings in Hilbert spaces," Journal of Mathematical Analysis and Applications, vol. 318, no. 1, pp. 43-52, 2006.

[12] W. Takahashi, N.-C. Wong, and J.-C. Yao, "Two generalized strong convergence theorems of Halpern's type in Hilbert spaces and applications," Taiwanese Journal of Mathematics, vol. 16, no. 3, pp. 1151-1172, 2012.

[13] H. Zhou, "Strong convergence of an explicit iterative algorithm for continuous pseudo-contractions in Banach spaces," Nonlinear Analysis. Theory, Methods \& Applications A. Theory and Methods, vol. 70, no. 11, pp. 4039-4046, 2009.

[14] G. L. Acedo and H.-K. Xu, "Iterative methods for strict pseudocontractions in Hilbert spaces," Nonlinear Analysis. Theory, Methods \& Applications A. Theory and Methods, vol. 67, no. 7, pp. 2258-2271, 2007.

[15] D. R. Sahu, H.-K. Xu, and J.-C. Yao, "Asymptotically strict pseudocontractive mappings in the intermediate sense," Nonlinear Analysis. Theory, Methods \& Applications A. Theory and Methods, vol. 70, no. 10, pp. 3502-3511, 2009.

[16] G. L. Acedo and H.-K. Xu, "Iterative methods for strict pseudocontractions in Hilbert spaces," Nonlinear Analysis. Theory, Methods \& Applications A. Theory and Methods, vol. 67, no. 7, pp. 2258-2271, 2007.

[17] L. C. Ceng, A. Petruşel, and J.-C. Yao, "Strong convergence of modified implicit iterative algorithms with perturbed mappings for continuous pseudocontractive mappings," Applied Mathematics and Computation, vol. 209, no. 2, pp. 162-176, 2009.
[18] L. C. Ceng, Q. H. Ansari, and J. C. Yao, "Strong and weak convergence theorems for asymptotically strict pseudocontractive mappings in intermediate sense," Journal of Nonlinear and Convex Analysis, vol. 11, no. 2, pp. 283-308, 2010.

[19] G. Marino and H.-K. Xu, "Weak and strong convergence theorems for strict pseudo-contractions in Hilbert spaces," Journal of Mathematical Analysis and Applications, vol. 329, no. 1, pp. 336-346, 2007.

[20] H. Zegeye, N. Shahzad, and M. A. Alghamdi, "Minimumnorm fixed point of pseudocontractive mappings," Abstract and Applied Analysis, vol. 2012, Article ID 926017, 15 pages, 2012.

[21] H. Zegeye, N. Shahzad, and T. Mekonen, "Viscosity approximation methods for pseudocontractive mappings in Banach spaces," Applied Mathematics and Computation, vol. 185, no. 1, pp. 538-546, 2007.

[22] H. Zhou, "Convergence theorems of fixed points for $\lambda$-strict pseudo-contractions in Hilbert spaces," Nonlinear Analysis. Theory, Methods \& Applications A. Theory and Methods, vol. 69, no. 2, pp. 456-462, 2008.

[23] L.-C. Zeng, N.-C. Wong, and J.-C. Yao, "Strong convergence theorems for strictly pseudocontractive mappings of BrowderPetryshyn type," Taiwanese Journal of Mathematics, vol. 10, no. 4, pp. 837-849, 2006.

[24] F. E. Browder and W. V. Petryshyn, "Construction of fixed points of nonlinear mappings in Hilbert space," Journal of Mathematical Analysis and Applications, vol. 20, pp. 197-228, 1967.

[25] C. E. Chidume and S. A. Mutangadura, "An example of the Mann iteration method for Lipschitz pseudocontractions," Proceedings of the American Mathematical Society, vol. 129, no. 8, pp. 2359-2363, 2001.

[26] Q.-B. Zhang and C.-Z. Cheng, "Strong convergence theorem for a family of Lipschitz pseudocontractive mappings in a Hilbert space," Mathematical and Computer Modelling, vol. 48, no. 3-4, pp. 480-485, 2008.

[27] P. E. Maingé, "Strong convergence of projected subgradient methods for nonsmooth and nonstrictly convex minimization," Set-Valued Analysis, vol. 16, no. 7-8, pp. 899-912, 2008.

[28] H.-K. Xu, "Iterative algorithms for nonlinear operators," Journal of the London Mathematical Society, vol. 66, no. 1, pp. 240-256, 2002. 


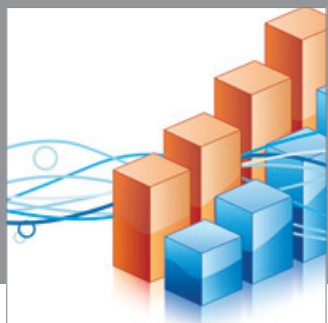

Advances in

Operations Research

mansans

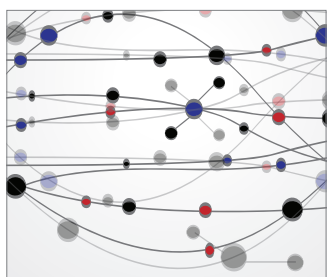

The Scientific World Journal
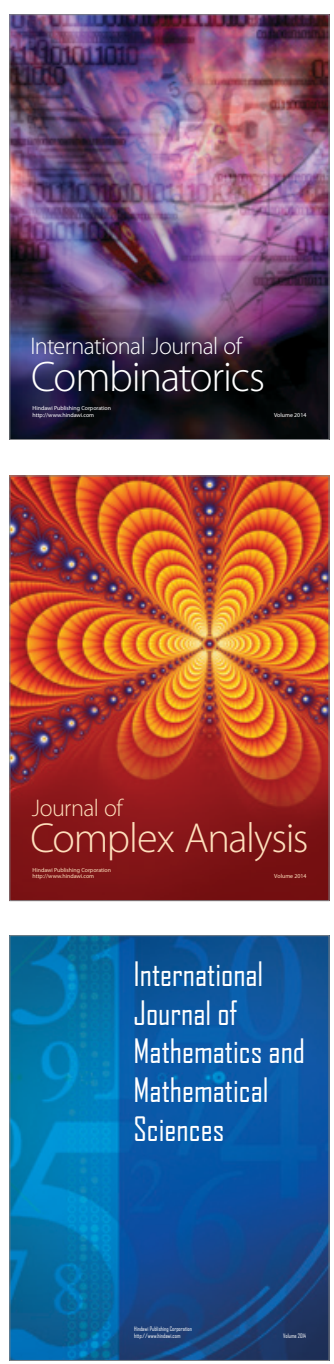
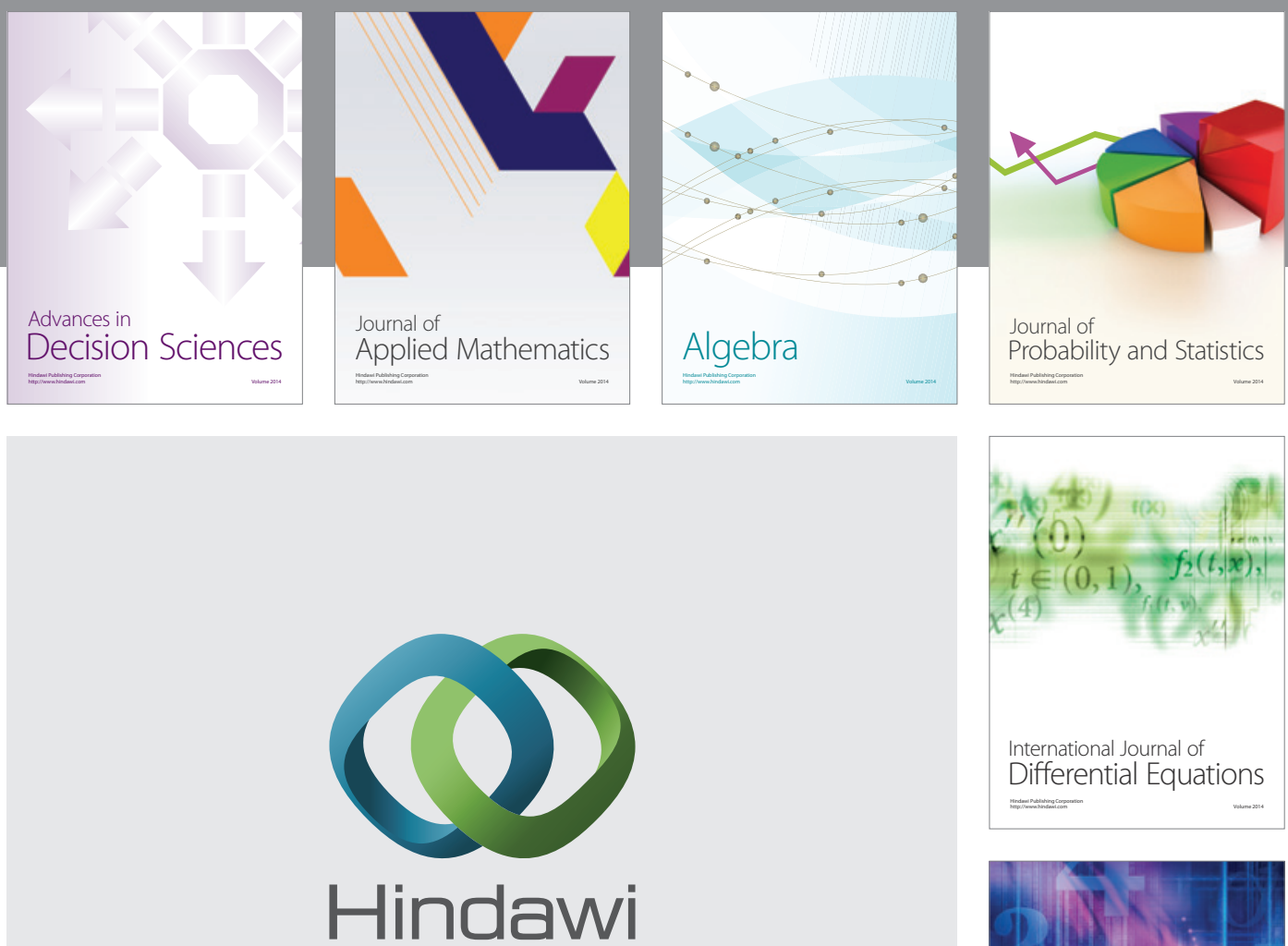

Submit your manuscripts at http://www.hindawi.com
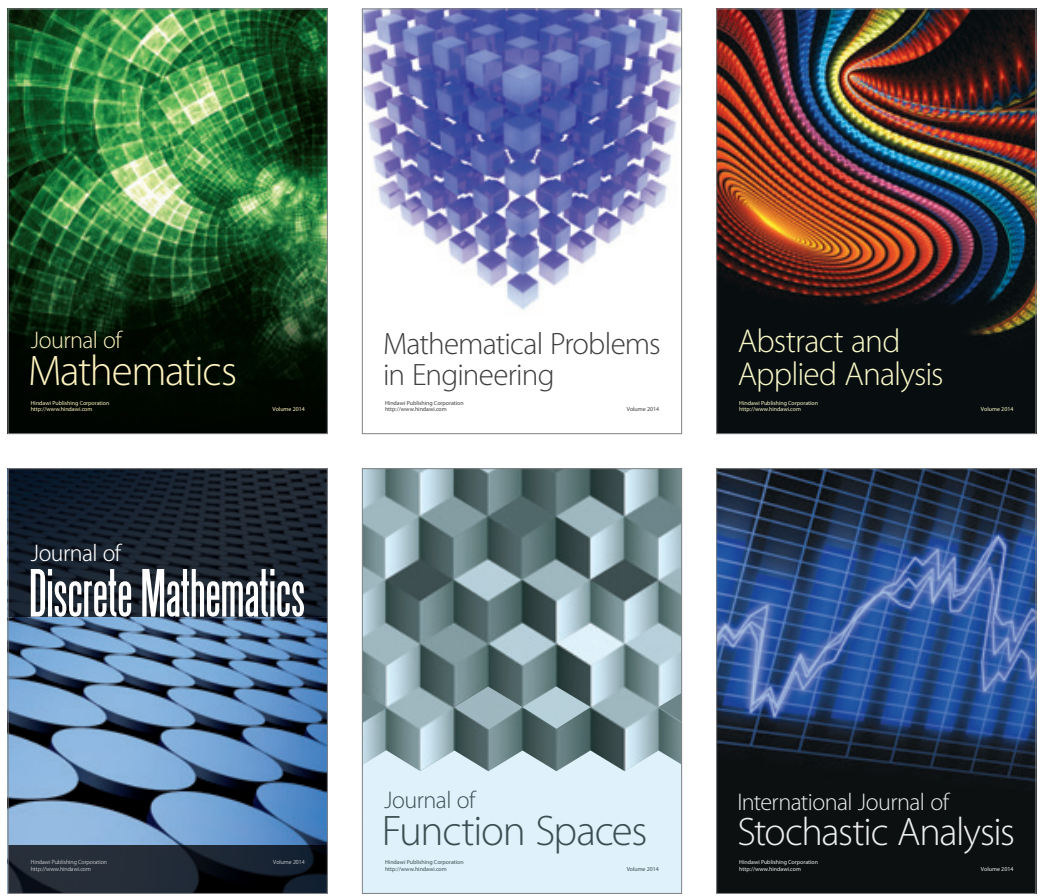

Journal of

Function Spaces

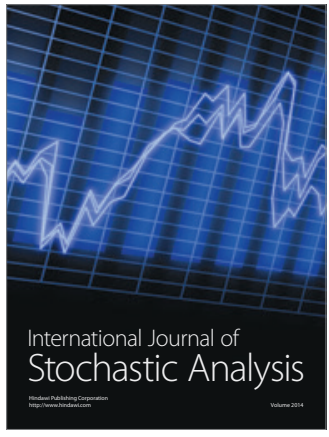

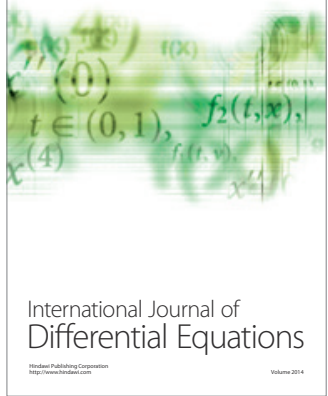
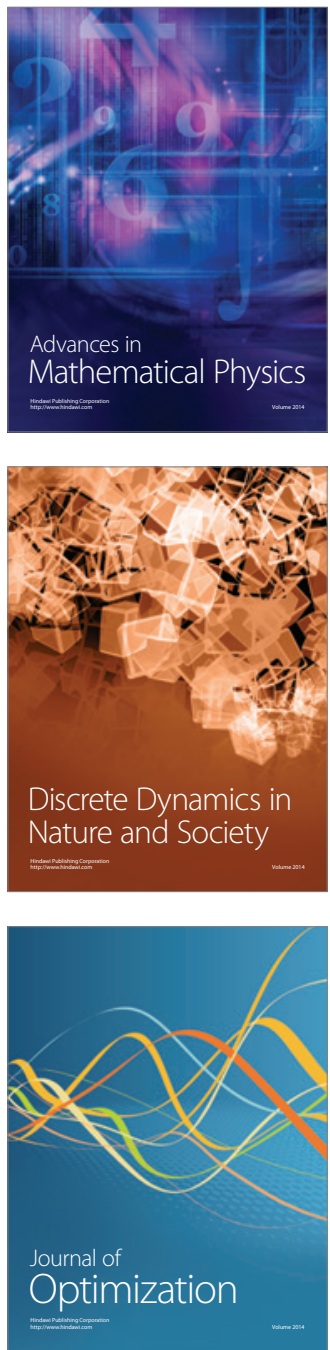